\title{
The variable clinical manifestations of ulnar neuropathies at the elbow
}

\author{
JOHN D STEWART \\ From the Division of Neurology, The Montreal General Hospital, Montreal, Quebec, Canada
}

SUMMARY In twenty-five cases of ulnar neuropathy at the elbow, the involvement of the fibres from three sensory and to four motor branches were examined clinically and, where possible, electrophysiologically. Of the sensory fibres, those from the terminal digital nerves were most commonly involved. The fibres to the hand muscles were much more frequently involved than those to the forearm muscles. These findings suggest that in ulnar neuropathies at the elbow there is variable damage to the fascicles within the nerve.

Ulnar neuropathies at the elbow frequently produce abnormalities in the small muscles of the hand while sparing the forearm muscles. ${ }^{1-10}$ It is less well known that the three sensory branches of the ulnar nerve, the terminal digital, dorsal ulnar cutaneous and palmar cutaneous branches, may also be variably involved. These findings have obvious clinical and electrodiagnostic relevance, and also raise the question of selective vulnerability of specific fascicles within the nerve. A prospective study was performed to investigate these patterns in more detail.

\section{Methods}

Twenty-four patients with clinical symptoms and signs of ulnar neuropathy in 25 arms were studied prospectively using a standardised protocol.

CLINICAL PROTOCOL

This included details of the following:

History

Onset (sudden or gradual); trauma (old, new; details of trauma); habitual elbow leaning; occupational repeated elbow flexion and extension; habitual elbow flexion in sleep; recent general anaesthetic. Symptoms: Paraesthesias; pain; weakness and/or clumsiness of the hand. Associated medical conditions: diabetes mellitus, chronic renal failure, rheumatoid or other arthritis, hypothyroidism, known cervical spondylosis.

Address for reprint requests: Dr JD Stewart. Montreal General Hospital. 1650 Cedar Avenue. Montreal. Quehec. Canada H3G IA4.

Received 6 August 1985 and in revised form 20 January 1986. Accepted 25 January 1986.
EXAMINATION

This included the following:

Elbow

Deformity: limitation of flexion or extension; excessive carrying angle; prolapsing nerve; the presence or absence of Tinel's sign.

\section{Motor}

Power was assessed in the deltoid, biceps, triceps, pronator teres, flexor carpi ulnaris (FCU), flexor digitorum profundus (FDP), flexor pollicis longus, abductor digiti minimi (ADM), first dorsal interosseous (FDI), the other interossei, the lumbrical and abductor pollicis brevis muscles.

\section{Sensory}

Light touch and pinprick sensation were assessed in the cutaneous distribution of the terminal digital (TD), dorsal cutaneous (DC), palmar cutaneous (PC), branches of the ulnar nerve (fig 1 ), and in the distribution of the median and radial nerves.

\section{Legs}

Power was assessed in the tibialis anterior and gastrocnemius muscles. Pinprick and vibration were examined in the toes; ankle reflexes were examined.

ELECTRODIAGNOSTIC PROTOCOL

Motor conduction studies

Surface disc electrodes were used to record simultaneously in two channels from ADM and FDI muscles. The ulnar nerve was stimulated supramaximally at the wrist and then at each of seven points, $2 \mathrm{~cm}$ apart, spanning the elbow ("inching" technique.). ${ }^{6}$ The distance between the most proximal and most distal stimulation sites across the elbow was therefore $12 \mathrm{~cm}$. The elbows were kept slightly flexed at an angle of about $150^{\circ}$ between the upper arm and forearm during the test, and the wrists were held fixed with a plastic splint with 

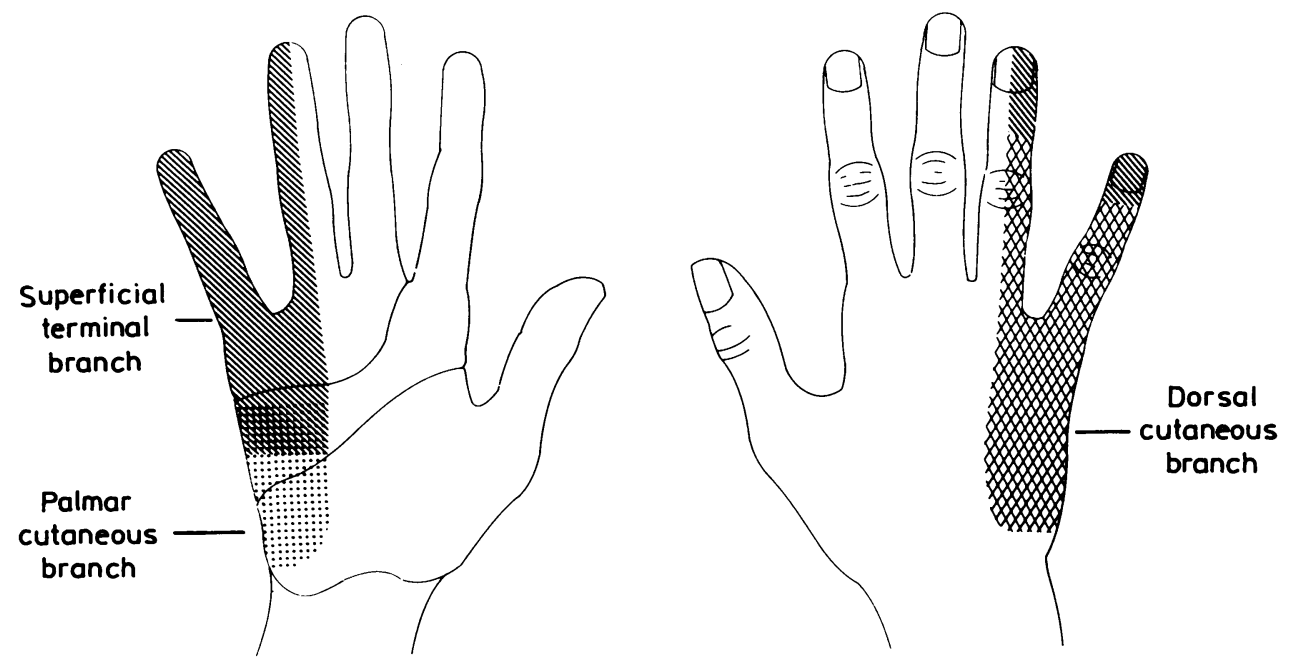

Fig 1 The cutaneous distribution of the three sensory branches of the ulnar nerve.

adjustable straps. The latencies (to initial deflection) and amplitudes (peak to peak) of the compound motor action potentials from FDI and ADM were measured. Conduction velocities across the elbows, from below the elbows to the wrist, and from the wrist to the ADM and FDI muscles were then calculated. The median nerve was also stimulated at the elbow and wrist while recording from ADM and FDI muscles to determine if a median-ulnar nerve (Martin-Gruber) anastamosis was present; four patients with probable Martin-Gruber anastamoses were excluded from the study.

Sensory conduction studies

The terminal digital branches of the 5th digit were stimulated using ring electrodes, with recording from disc electrodes over the ulnar nerve at the wrist. The technique used for the dorsal ulnar cutaneous (DC) nerve study was that described by Jabre: ${ }^{11}$ disc electrodes were placed over the nerve where it courses over the dorsal surface of the fifth metacarpal bone, and the nerve was stimulated where it winds around the lateral aspect of the humerus about $2 \mathrm{~cm}$ proximal to the wrist crease. Latencies were taken to the onset of the major negative deflection and conduction velocities were calculated using distance measurements; amplitudes were measured from peak to peak. Sensory studies were also done on the opposite hand.

\section{Electromyography}

The FCU, ulnar innervated part of flexor digitorum profundus $\left(\mathrm{FDP}_{\mathrm{u}}\right), \mathrm{ADM}, \mathrm{FDI}$ and $\mathrm{APB}$ muscles were examined with a concentric needle electrode at rest and during volitional contraction. Electrode placements were those described by Delagi and Perotto. ${ }^{12}$ The correct needle placement for the $\mathrm{FDP}_{\mathrm{u}}$ muscle was verified by having the patient flex just the terminal phalanx of the 5th digit.

CRITERIA FOR THE DIAGNOSIS OF ULNAR

NEUROPATHY AT THE ELBOW

The diagnosis of probable ulnar neuropathy was made when there was numbness and/or weakness in the distribution of one or more of the major branches of the ulnar nerve, with no abnormalities of other peripheral nerves in that limb. Clinically definite ulnar neuropathies at the elbow were those in which the sensory loss included the palmar and/or dorsal cutaneous branches (because these branches arise in the forearm and do not pass through Guyon's canal), or when

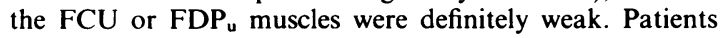
with motor and sensory signs restricted to branches arising at or distal to the wrist were included as definite ulnar neuropathies at the elbow if either $(a)$ motor nerve conductions from the wrist to FDI or ADM muscles were normal, while conduction block (see below) was present when the nerve was stimulated above the elbow, and/or (b) EMG studies showed abnormalities in either or both the FCU and/or FDP $_{\mathrm{u}}$. These criteria were also used for including two patients with purely sensory symptoms and signs in the distribution of the ulnar nerve.

\section{ELECTROPHYSIOLOGICAL CRITERIA}

(a) Motor conduction studies These were considered abnormal when the amplitudes of the compound motor action potentials from FDI and/or ADM were reduced by at least $20 \%$ following stimulation of the nerve above the elbow compared with at the wrist, that is, conduction blocking. (In 40 ulnar nerves of 20 normal persons, the maximum amplitude decrement when stimulating the ulnar nerve above the elbow compared with at the wrist was $10 \%$.) Conduction velocities across the elbow were not used as a criterion of focal conduction slowing because of the wide range of values found in normal subjects. (In 40 ulnar nerves of 20 normal persons, the motor conduction velocities over a $12 \mathrm{~cm}$ distance across the elbow ranged from $39-100 \mathrm{~m} / \mathrm{s}$ (recording from FDI) and 43-92 m/s (recording from ADM). Conduction velocities from both ulnar nerves in the same person could vary by as much as $40 \mathrm{~m} / \mathrm{s}$. These inaccuracies are probably the result of several factors: $(a)$ current spread from the stimulating electrodes may depolarise the nerve some variable distance from the electrode, $(b)$ the laxity of the skin around the elbow can cause the electrode 
Table 1 Causes in 25 ulnar neuropathies

True tardy palsy*

Recent elbow trauma without fracture

Habitual leaning on elbow

Habitual elbow flexion in sleep ${ }^{15-17}$

Recent general anaesthetic

Occupational repetitive flexion/extension

Prolapsing nerve†

Diabetes mellitus

One putative cause

Several putative causes

No apparent cause

*Old fracture with deformity ${ }^{1314}$

†All of mild degree ${ }^{18}$

position to move even when careful skin markings have been made, $(c)$ the relatively short distance $(12 \mathrm{~cm})$ between the proximal and distal stimulation sites means that small errors in the measurement of this distance produce relatively large changes in the calculated motor conduction velocities).

(b) Sensory conduction studies The TD sensory action

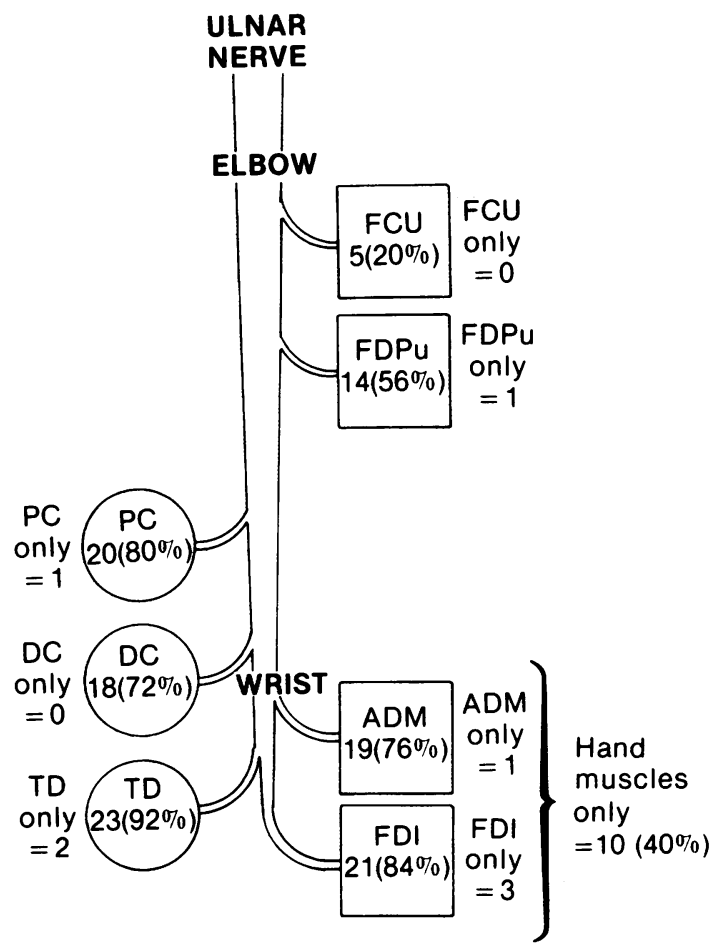

Fig 2 Clinical abnormalities in the distribution of 3 sensory and 4 motor branches of the ulnar nerve. "Only" is used to denote the number of patients in whom a single sensory area was involved; and to denote those patients with weakness of only one of the four muscles. $P C=$ palmar cutaneous,

$D C=$ dorsal cutaneous, $T D=$ terminal digital branches,

$F C U=$ flexor carpi ulnáris, $F D P_{\mathrm{i}}=$ flexor digitorum

profundus, $F D I=$ first dorsal interosseous, $A D M=$ abductor digiti minimi. potential was judged to be abnormal when it was less than $8 \mu \mathrm{v}$. (Our normal values [35 nerves]: Amplitude mean $=$ $21.3 \mu \mathrm{v}$; mean $-2 \mathrm{SD}=8.4)$. The DUC sensory action potential was considered abnormal when less than $5.5 \mu \mathrm{v}$ (Our normal values [ 35 nerves]: Amplitude mean $=19.4 \mu \mathrm{v}$ mean $-2 \mathrm{SD}=5 \cdot 5 \mu \mathrm{v})$. (See also references 4,11 ).

(c) $E M G$ Axonal destruction in the nerve fibres supplying a muscle was considered to have occurred when, at the minimum, several runs of fibrillation potentials and/or positive sharp waves were recorded from that muscle. Other criteria of "neurogenic" changes (size, configuration, and recruitment patterns of motor unit potentials) were not used because of the subjective nature of the interpretation of these findings.

\section{Results}

Twenty-four patients with 25 ulnar neuropathies were studied. The causes are summarised in table 1.

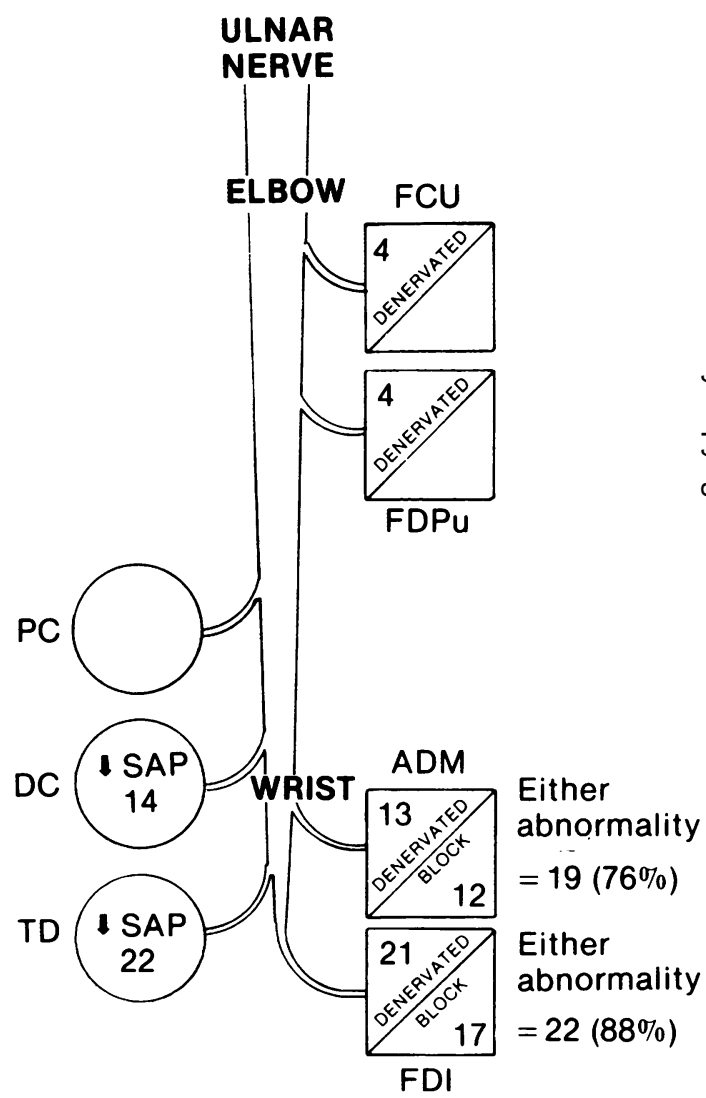

Fig 3 Electrophysiological abnormalities in the nerve fibres of 2 sensory and 4 motor branches of the ulnar nerve. $\downarrow S A P=$ diminished sensory action potential amplitude. For involvement of muscles, the number of patients in whom denervation ("denervated") was present is shown for each muscle, while in addition, the numbers of muscles in which conduction blocking ("block") was found are also shown. 
Table 2 Dencrvation and conduction hlocking in ADM and FDI muscles (25 ulnar neuropathie's).

\begin{tabular}{lc}
\hline Ahductor digiti minimi & \\
Clinical weakness & $19(76 \%)$ \\
* Dencrvation & 13 \\
* Blocking & 12 \\
Denervation only: no blocking & 7 \\
Blocking only; no denervation & 6 \\
Both & 6 \\
Either denervated or blocked or both & $19(76 \%)$ \\
Firsl dorsal inlerossseous & \\
Clinical weakness & $21(84 \%)$ \\
Denervation & 21 \\
Blocking & 17 \\
Denervation only: no blocking & 5 \\
Blocking only: no denervation & 1 \\
Both & 16 \\
Either denervated or blocked or both & $22(88 \%)$ \\
\hline
\end{tabular}

*See text for criteria.

\section{Clinical findings}

Figure 2 shows the motor and sensory abnormalities. The frequency of sensory involvement was greatest in TD, less in PC, and least in DUC. The frequency of motor involvement was greatest in FDI, less in ADM, still less in FDP $\mathrm{F}_{\mathrm{u}}$, and least in FCU.

\section{Electrophysiological abnormalities}

Figure 3 shows the electrophysiological abnormalities detected in two sensory branches and four muscles. Denervation and conduction blocking were more frequently found in the FDI than ADM muscles (table 2). In a few patients either of these muscles was involved (as judged clinically or electrophysiologically) independently of the other muscle. The results of the "inching" study will be described elsewhere.

\section{Specific patients}

$R A C$ This man developed a true tardy ulnar neuropathy 30 years after fracturing the distal humerus. There was sensory loss in the distribution of all three

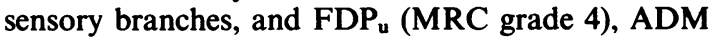
(grade 2), FDI (grade 3) were weak, but FCU was normal. ADM and FDI were denervated while FCU and $\mathrm{FDP}_{\mathrm{u}}$ were not. At operation, there was no evidence of nerve compression within the cubital tunnel: the nerve was not swollen proximal to the flexor carpi ulnaris aponeurosis, and the cubital tunnel was roomy when tested with a probe even when the elbow was flexed. ${ }^{16}$ The branch to FCU arose from the usual position just distal to the medial epicondyle. About $2 \mathrm{~cm}$ proximal to the medial epicondyle the nerve appeared thin and discoloured but was not compressed by any abnormal structures. Intraoperative recordings showed a highly localised conduction block at that level (fig 4). Thus a lesion of the ulnar nerve was confirmed to be about $4 \mathrm{~cm}$ proximal to the origin of the branch to FCU muscle, yet this
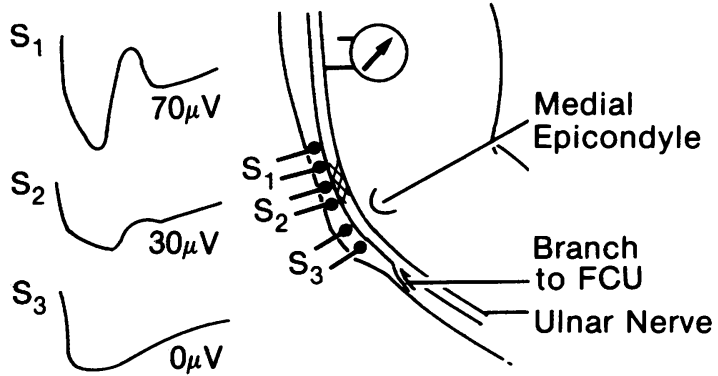

Fig 4 Patient RAC. Recording was from a needle electrode placed in the epineurium of the ulnar nerve $8 \mathrm{~cm}$ proximal to the epicondyle. Stimulation sites were $3 \mathrm{~cm}\left(S_{1}\right)$ and $1.5 \mathrm{~cm}\left(S_{2}\right)$ proximal to the epicondyle, and adjacent to the epicondyle $\left(S_{3}\right)$. The nerve appeared abnormal at the cross-hatched area. The branch to the unaffected flexor carpi ulnaris (FCU) was identified well distal to the site of the lesion. Stimulation at $S_{1}$ produced a compound nerve action potential (the mixed activity of sensory fibres conducting orthodromically and motor fibres conducting antidromically) of $70 \mu \mathrm{V}$. Stimulating at the site of the lesion $\left(S_{2}\right)$ and on the opposite side of the lesion $\left(S_{3}\right)$ produced a reduced and absent compound nerve action potential respectively, showing conduction blocking across the nerve lesion.

muscle was totally unaffected by the nerve lesion. $M A R$ This patient presented with numbness in the distribution of the TD branch only. There was a history of habitual leaning on the elbow and elbow flexion during sleep. Examination showed the ulnar nerve to be tender in the condylar groove with a positive Tinel's sign, and the restricted sensory loss was confirmed. All muscles were normal except for mild weakness of FDI. The distribution of the sensory and motor signs indicate that this ulnar nerve lesion could be in the hand distal to the origins of the branches to the hypothenar muscles. However, the electrophysiological studies showed a conduction block at the elbow in the nerve fibres supplying FDI, and also denervation of the $\mathrm{FDP}_{\mathrm{u}}$, demonstrating that the site of the lesion was the elbow. FDI, but not ADM, was also denervated.

STG This patient also presented with only TD distribution sensory loss. All of the small muscles of the hand innervated by the ulnar nerve were weak. This suggested a lesion of the ulnar nerve at the wrist, proximal to the motor branches to the hypothenar muscles (as for example, in Guyon's canal). ${ }^{19}$ However, the motor conduction velocities from the wrist to the FDI and ADM muscles were normal while there was a conduction block across the elbow when recording from FDI. These findings showed that the site of the ulnar neuropathy was the elbow.

\section{Discussion}

Although nerve lesions are customarily localised by 
considering the longitudinal anatomy of the nerve and its branches (as in fig 2), this approach has its pitfalls. For example, ulnar nerve lesions at the elbow, clearly proximal to the sites at which branches to the flexor carpi ulnaris muscle usually arise ${ }^{20}$ often spare this muscle. The branches to flexor digitorum profundus, the palmar cutaneous and the dorsal cutaneous branches arise at considerable distances distal to the medial epicondyle, and the fibres to these branches may be involved or spared in lesions of the nerve at the elbow. The only reasonable explanation for this is differential involvement of fascicles within the nerve. The present study shows that the fascicles most frequently affected are those that contain the nerve fibres from the terminal digital sensory branches and those to the small muscles of the hand, particularly the FDI. Confirmation of this "fascicular phenomenon" comes from patient RAC: preoperative EMG studies showed that ADM and FDI muscles were denervated, but that FCU and FDP $_{u}$ were not. At operation the branches to FCU were seen to arise $2 \mathrm{~cm}$ distal to the medial epicondyle, while intra-operative nerve conduction studies showed that the damage was $2 \mathrm{~cm}$ proximal to the epicondyle. This confirms that a proximal lesion may involve certain fascicles (to ADM and FDI) while sparing others (to FCU and FDP ). .

Two (patients MAR and STG described above) of the 25 ulnar neuropathies would have been, on the clinical pattern of motor and sensory abnormalities, localised to the wrist or hand. However, the electrodiagnostic studies showed that the site of the focal neuropathy was at the elbow. These patients illustrate the clinical relevance of this selectivity of fascicular involvement. This difficulty in localisation was clearly stated by Osborne in 1959:1 "selective involvement of the hand muscles ... creates difficulty in diagnosis, as the picture may become confused with neuritis at wrist level ..."

An early description of fascicular phenomena was that of the Dejerines and Mouzon in 1915, who studied traumatic lesions of forearm nerves in French soldiers wounded in World War I. ${ }^{21}$ They described these "dissociated syndromes" thus: "Each fascicle of each nerve possesses, in effect, an individuality. It can be injured independently of its neighbours, or to a different degree. It can also be spared while the other fascicles are injured to a greater or lesser extent". There are, in fact, many familiar examples of selective involvement of parts of peripheral nerves. In sciatic neuropathies, the lateral trunk (which forms the common peroneal nerve) is often more severely involved than the medial trunk (which forms the tibial nerve), and therefore a sciatic neuropathy may present with a foot-drop, masquerading as a common peroneal palsy. ${ }^{223}$ Trojaborg has pointed out the variability of involvement of the sensory fibres and various motor fibres within the radial nerve in Saturday night palsies: branches to the brachioradialis muscle and/or the sensory branch may be completely spared in lesions well above their sites of origin. ${ }^{24}$ In the thoracic outlet syndrome caused by a fibrous band distorting the lower trunk of the brachial plexus, there is often selective involvement of the intrinsic hand muscles that derive their innervation from the trunk; the median nerve-innervated abductor pollicis brevis is usually exclusively or markedly more affected than the ulnar nerve-innervated intrinsic muscles. ${ }^{25} \mathrm{~A}$ striking example of a proximal partial lesion masquerading as an isolated distal lesion is the report of a patient with brachial neuritis with weakness initially restricted to the muscles supplied by the anterior interosseous nerve. ${ }^{26}$ Unequal ultrastructural involvement of fascicles has been described in experimental focal neuropathies in animals. ${ }^{2728}$ Detailed microscopic examination of human ulnar nerves at the elbow has also shown variable involvement amongst fascicles. ${ }^{29}$

The detailed anatomy of human peripheral nerve fascicles is controversial. It has been generally accepted, largely from the work of Sunderland, ${ }^{20}$ that the fascicles in the distal part of the nerve comprise fibres from individual branches of the nerve (simple fascicles), while proximal fascicles contain increasing mixtures of fibres from the different branches (compound fascicles). This mixing is said to result from many intercommunications between fascicles. In Sunderland's single dissected specimen of an ulnar nerve it consisted mainly of compound fascicles at the elbow. However, Jabaley $e t$ al found that the fascicles going to FCU and FDP muscles, and that from the dorsal ulnar cutaneous nerve, were quite distinct at that level. ${ }^{30}$ Recent microneurographic experiments in the median nerve also suggest that there may be less fibre intermingling between fascicles than previously thought. ${ }^{31}$ Regardless of the details of the contents of the fascicles, in the region of the elbow the nerve fibres from the terminal digital sensory branch and to the small muscles of the hand lie deeply in the nerve, adjacent to bone. It has been suggested that this position renders these fascicles more susceptible to damage from external pressure. ${ }^{20}$

Another explanation for the current findings can be put forward. The sensory and motor branches most affected are those that arise most distally from the nerve. Proximal pressure could compromise axoplasmic flow which would then preferentially affect the longest fibres in the nerve, resulting in a dying-back phenomenon in the branches to the skin and muscles of the hand. This is unlikely for several reasons: $(a)$ in other focal neuropathies in which selective muscle involvement/sparing has been noted (radial and com- 
mon peroneal nerves), the most distal muscles are not predominantly affected, ${ }^{2332}(b)$ some patients in this study had involvement of one of the major ulnar nerve-innervated intrinsic hand muscles while the other was unaffected, and both are supplied by distal branches of the nerve. However, a dying-back phenomemon probably docs occur to some degree in compressive focal neuropathies: in the carpal tunnel syndrome and radiculopathies, the sensory abnormalitics are usually present in the most distal part of the cutaneous area supplied by that nerve or spinal nerve root.

Numerous experimental studies of focal compression neuropathies has cstablished that the microscopic sequelac range from focal demyelination to axonal brcakdown. In the clectromyography laboratory, these processes are easiest to evaluate in motor nerve fibres: focal demyelination can be detected by conduction slowing and blocking, while axonal degeneration produces denervation potentials in affected muscles. The present study shows that varying degrees of these two basic processes can occur in different fascicles within the same nerve. Considcrably more information could probably be obtained if EMG and nerve conduction studies were performed on other ulnar nerve-innervated muscles. In sensory fascicles, conduction blocking could be examined by recording with needle electrodes at the wrist and above the elbow. ${ }^{4}$

In conclusion, this present study confirms and expands on previous observations that ulnar neuropathies at the elbow produce variable involvement of sensory and motor fascicles. Certain fascicles are more susceptible to damage than others, notably the three sensory fascicles (particularly TD), and the fascicles to the intrinsic hand muscles (particularly FDI). These selective patterns of fascicular involvement add a dimension of difficulty to the localisation of peripheral nerve lesions. It is not sufficient for localisation to be based entirely on longitudinal anatomy and branching patterns of nerves and plexuses. A knowledge of these fascicular phenomena is also important to the clectromyographer: abnormalities are more frequently detected in the FDI than the ADM muscle, and therefore FDI is a better muscle to use in patients suspected of having ulnar neuropathies. However, studying both muscles further increases the diagnostic yicld.

The intra-operative studies were performed in conjunction with Dr PM Richardson. Mrs Maria Hall and Mrs Arlene Berg provided expert technical and secretarial help.

This work was presented at the meeting of the American Academy of Neurology, Boston 1984.

\section{References}

1 Osborne G. Ulnar neuritis. Postgrad Med 1959;35:392-6.

2 Gilliatt RW, Thomas PK. Changes in nerve conduction with ulnar lesions at the elbow. $J$ Neurol Neurosurg Psychiatry 1960;23:312-20.

3 Vanderpool DW, Chalmers J, Lamb DW, Whiston TB. Peripheral nerve lesions of the ulnar nerve. $J$ Bone Joint Surg 1968;50B:792-801.

4 Payan J. Electrophysiological localization of ulnar nerve lesions. J Neurol Neurosurg Psychiatry 1969;32: 208-20.

5 Eisen A. Early diagnosis of ulnar nerve palsy. Neurology 1974:24:256-62.

6 Miller RG. The cubital tunnel syndrome: diagnosis and precise localization. Ann Neurol 1979;6:56-9.

7 Jabre JF, Wilbourn AJ. The EMG findings in 100 consecutive ulnar neuropathies. Acta Neurol Scand 1979;60:91.

8 Laha RK, Panchal PD. Surgical treatment of ulnar neuropathy. Surg Neurol 1979;11:393-8.

9 Chan RC, Paine KWE, Varughese G. Ulnar neuropathy at the elbow: comparison of simple decompression and anterior transposition. Neurosurgery 1980;7:545-50.

10 Craven PR, Green DP. Cubital tunnel syndrome. J Bone Joint Surgery 1980;62A:986-9.

11 Jabre JF. Ulnar nerve lesions at the wrist: new technique for recording from the sensory dorsal branch of the ulnar nerve. Neurology 1980;30:873-6.

12 Delagi EF, Perotto A. Anatomic Guide for the Electromyographer. 2nd ed. Springfield, Illinois. Charles C Thomas, 1980.

13 Panas P. Sur une cause peu connue de paralysie du nerf cubital. Arch Gén Méd 1878;2:5-20.

14 Mouchet A. Paralysies tardives du nerf cubital à la suite des fractures du condyle externe de l'humerus. J Chirurg (Paris) 1914;12:435-56.

15 Buzzard FF. Some varieties of traumatic and toxic ulnar neuritis. Lancet 1922;1:317-9.

16 Feindel W, Stratford J. The role of the cubital tunnel in tardy ulnar palsy. Can J Surg 1958;1:287-300.

17 Apfelberg DB, Larson JL. Dynamic anatomy of the ulnar nerve at the elbow. Plast Reconstr Surg 1973:51:76-81.

18 Childress HM. Recurrent ulnar nerve dislocation at the elbow. J Bone Joint Surg 1956;38A:978-84.

19 Shea JD, McClain EJ. Ulnar nerve compression syndromes at and below the wrist. $J$ Bone Joint Surg 1969;51 A:1095-103.

20 Sunderland S. Nerves and Nerve Injuries. Edinburgh: Churchill Livingstone, 1978.

21 Dejerine J, Dejerine A, Mouzon J. Les lésions de gros troncs nerveux des membres par projectiles de guerre. Presse Med 1915;40:321-8.

22 Stookey B. Gunshot wounds of peripheral nerves. Surg Gynecol Obstet 1916;23:639-56.

23 Sunderland $S$. The relative susceptibility to injury of the medial and lateral popliteal divisions of the sciatic nerve. Br J Surg 1953;41:2-4.

24 Trojaborg W. Rate of recovery in motor and sensory fibres of the radial nerve: clinical and electro- 
physiological aspects. J Neurol Neurosurg Psychiatry 1970;33:625-38.

25 Gilliatt RW, Willison RG, Dietz V, Williams IR. Peripheral nerve conduction in patients with a cervical rib and band. Ann Neurol 1978;4:124-9.

26 Rennels GD, Ochoa J. Neuralgic amyotrophy manifesting as anterior interosseous nerve palsy. Muscle Nerve 1980;3:160-4.

27 Fullerton PM, Gilliatt RW. Pressure neuropathy in the hind foot of the guinea-pig. J Neurol Neurosurg Psychiatry 1967;30:18-25.

28 Aguayo A, Nair CPV, Midgley R. Experimental progressive compression neuropathy in the rabbit.
Arch Neurol 1971;24:355-64.

29 Neary D, Eames RA. The pathology of ulnar nerve compression in man. Neuropathol Appl Neurobiol 1975:1:69-88.

30 Jabaley ME, Wallace WH, Heckler FR. Internal topography of major nerves of the forearm and hand: a current view. J Hand Surg 1980;5:1-18.

31 Schady W, Ochoa JL, Torebjork HE, Chen LS. Peripheral projections of fascicles in the human median nerve. Brain 1983;106:745-60.

32 Sourkes M, Stewart JD. Patterns of fascicular involvement in common peroneal neuropathies. Can J Neurol Sci 1984;11:330. 